\title{
Determination of Diagnostic Value of Toxoplasma gondii Recombinant ROP2 and ROP4 Antigens in Mouse Experimental Model
}

\author{
JUSTYNA GATKOWSKA ${ }^{1 *}$, BOŻENA DZIADEK ${ }^{1}$, ANNA BRZOSTEK ${ }^{2}$, JAROSŁAW DZIADEK ${ }^{2}$, \\ KATARZYNA DZITKO ${ }^{1}$ and HENRYKA DŁUGOŃSKA ${ }^{1}$ \\ ${ }^{1}$ Department of Immunoparasitology, University of Łódź, Poland \\ ${ }^{2}$ Centre for Medical Biology, Polish Academy of Sciences, Łódź, Poland
}

Received 13 September 2009, revised 28 December 2009, accepted 16 January 2010

Abstract

\begin{abstract}
The aim of this study was to test the potential diagnostic usefulness of recombinant Toxoplasma gondii rhoptry antigens, ROP2 and ROP4, with respect to toxoplasmosis detection and infection phase distinction in laboratory mouce by determining specific serum $\operatorname{IgM}$ and IgG antibodies with the use of indirect ELISA technique. The mice antibody response to ROP antigens was significantly higher in the IgM than in the IgG class with the peak on the turn of acute and latent infection, whereas the response to recombinant SAG1 antigen, used as control, revealed preferential synthesis of IgG antibodies with the highest absorbance values measured during latent toxoplasmosis.
\end{abstract}

K e y w ord s: Toxoplasma gondii, antibody detection, experimental toxoplasmosis, inbred mice, recombinant antigens.

Despite many years of research, T. gondii infection one-step diagnostic methods based on IgM/IgG antibodies detection and level comparison may prove insufficient to define the phase of toxoplasmosis in certain cases, like pregnancy or immunodeficiency. Paradoxically, especially for these patients the determination of the exact invasion point is of great importance. Thereby, second step procedures are involved, which makes laboratory diagnostics both more expensive and more time-consuming (Montoya and Liesenfeld, 2004). Besides, most serological tests require native antigens of tachyzoites harvested from mice or cell cultures. Since constant quality and specificity of these antigens are not satisfactory, the use of recombinant antigens could abolish these disadvantages, lower production and purification costs and enable selection of antigens appropriate for the discrimination of toxoplasmosis phases. Several literature data reveal the potential usefulness of recombinant antigens for toxoplasmosis diagnostics (Aubert et al., 2000; Buffolano et al., 2005; Jacobs et al., 1999; Pietkiewicz et al., 2004) and even reflect attempts to employ them as tools for $T$. gondii invasion phase differentiation, however with discordant results (Ferrandiz et al., 2004; Nigro et al., 2003; Pfrepper et al., 2005).
The object of this study was to assess the diagnostic utility of two recombinant Toxoplasma rhoptry proteins ROP2 and ROP4, members of the prominent rhoptry protein family ROP2, for the recognition of the parasite invasion in mice by immunoenzymatic detection of IgM and IgG antibodies in immune sera. Experimental toxoplasmosis in inbred mouse strains is a widely accepted model to study the immune response to T. gondii and to pre-evaluate the diagnostic value of tested antigens. Rhoptry antigens are secreted from specialized apical organelles of T. gondii and they participate in the penetration and formation of the parasitophorous vacuole membrane (PVM) (Beckers et al., 1994; Carey et al., 2004). Moreover, ROP2 antigen is involved in the PVM - host cell organelle association (Sinai and Joiner, 2001) and contains T-cell epitopes recognized by a high percentage of Toxoplasma infected individuals (Saavedra et al., 1996). There are data concerning the potential use of this antigen for human toxoplasmosis diagnostics, however many of them focus only on IgG detection (de Souza Macre et al., 2009; Nigro et al., 2003; van Gelder et al., 1993) and besides, to our knowledge, this study describes the first attempt to evaluate the diagnostic utility of $T$. gondii ROP4 protein.

* Corresponding author: J. Gatkowska, Department of Immunoparasitology, University of Łódź, 90-237 Łódź, Banacha 12/16, Poland; phone: (+48) 4263543 55; fax: (+48) 4266558 18; e-mail: gatjus@biol.uni.lodz.pl 
For the cloning of recombinant antigens standard molecular biology protocols were used (Sambrook and Russell, 2001). Cloning of rop2 and rop 4 of $T$. gondii and expression of the respective recombinant proteins were described previously (Dziadek et al., 2009). Using the same procedure the inner fragment (882 bp) of T. gondii sagl (S76248) encoding 294 amino acids of $337 \mathrm{aa}$ whole protein (from 18aa to 311 aa, except leader and transmembrane domain sequences) was cloned and expressed in E. coli system.

The efficiency of the purification process was verified by $12 \%$ polyacrylamide gel SDS-PAGE and staining with Imperial Protein Stain (Pierce). The recombinant proteins migrated with the molecular masses of $38 \mathrm{kDa}, 35 \mathrm{kDa}$ and $31 \mathrm{kDa}$, respectively, in according with theoretical values based on the amino acid sequences. The amount of all purified recombinant proteins (ROP2, ROP4 and SAG1) was evaluated by Bradford technique. The total amount of expressed purified proteins yielded as follows: $72.8 \mathrm{mg}$ (ROP2), $160.0 \mathrm{mg}$ (ROP4) and $65.9 \mathrm{mg}$ (SAG1) from 11 of induced bacterial cultures.

In order to obtain mouse immune sera experimental toxoplasmosis was induced by intraperitoneal inoculation of 5 brain cysts of low virulent $T$. gondii DX strain (genotype II) into male BALB/c inbred mice 10 weeks of age. Toxoplasma cysts were obtained as described previously (Gatkowska et al., 2006). Briefly, the cysts were isolated from the brain of latently infected BALB/c mouse by gradient separation from mechanically homogenized brain tissues. All experimental procedures on mice were approved by the Local Ethics Commission in Łódź.

The immune sera were collected at three time points after primary $T$. gondii invasion: in the early acute phase (1 week post-infection), on the turn of the acute and chronic phase ( 3 weeks post-infection) and in the advanced latent phase of toxoplasmosis ( 9 weeks post-infection). Sera derived from uninfected animals of the same gender and age served as negative controls. All experimental groups comprised 10 animals.

Prepared purified recombinant $T$. gondii antigens were used as diagnostic antigens for the detection of specific anti-Toxoplasma antibodies in mouse sera by the use of indirect standard ELISA performed as described previously (Gatkowska et al., 2006). Serological MaxiSorp plates (NUNC) were coated with recombinant ROP2, ROP4 or SAG1 (control) antigen, at a concentration of $1 \mathrm{mg} / 50 \mu \mathrm{l} /$ well as determined in preliminary experiments. The immune and control mouse serum samples were diluted 1:100 and added in a final volume of $50 \mu \mathrm{l}$. The immunoenzymatic reaction was developed with goat anti-mouse $\operatorname{IgM}$ or IgG antibodies (Jackson ImmunoResearch) labe- led with HRP (secondary antibodies) and chromogen - 2,2'-azino-bis(3-ethylbenzothiazoline-6-sulfonic acid) diammonium salt (ABTS) (Sigma) at the concentration of $1 \mathrm{mg} / \mathrm{ml}$. All samples were run in duplicate and the results were presented as mean absorbance values \pm standard deviation. Additionally the cut-off values, expressed as the mean reactivity of negative sera +2 standard deviations, were calculated for tested recombinant antigens and statistical analysis was performed with the use of STATISTICA 5.0 PL software. Differences in U Mann-Whitney test were considered significant for $\mathrm{p}<0.05$.

Since the aim of this study was to determine whether recombinant rhoptry proteins ROP2 and ROP4 may serve as potentially useful antigens allowing both the detection of toxoplasmosis and the determination of the infection phase, the levels of specific anti-ROP2 and anti-ROP4 antibodies in mouse sera were tested with obtained rhoptry antigens and compared to the reactivity of sera with SAG1 protein. Mouse sera poorly recognized all recombinant antigens in early infection phase (1 week post-infection) both in IgM and IgG class as displayed in Fig. 1. On the turn of the acute and chronic phase (3 weeks postinfection) the level of specific anti-ROPs IgM antibodies rose considerably reaching the maximum value and was significantly higher when compared to the concentration in the chronic phase $(p<0.0003$ for ROP2 and $p<0.008$ for ROP4). The pattern of IgG response to ROP antigens was similar to that of IgM reactivity but the levels of IgG antibodies specific for both ROP2 and ROP4 were lower than IgM immunoglobulins concentration at the two tested time points after Toxoplasma challenge $(\mathrm{p}<0.0004)$. In contrast to ROP antigens, the response to SAG1 antigen revealed mainly the synthesis of IgG antibodies starting from the late acute phase ( 3 weeks post-infection), their concentration rose along with the infection time $(p<0.002$ for comparison of IgG levels 3 and 9 weeks after primary invasion) and in the chronic toxoplasmosis exceeded several times the amounts of antiROP IgG antibodies $(\mathrm{p}<0.00008)$.

It is known that the common approach to toxoplasmosis detection and phase differentiation based exclusively on $\operatorname{IgM}$ and $\operatorname{IgG}$ detection and comparison of concentration fails in some cases, which means that second-step diagnostic procedures are required. The most popular subsequent approach involves the determination of IgG avidity which may not be helpful in defining the current status of the invasion in immunocompromised individuals (Mechain et al., 2000) or in pregnant women treated with spiramycin which delays both production and avidity maturation of anti-Toxoplasma IgG antibodies (Meroni et al., 2009). Moreover, IgM antibodies, usually associated 

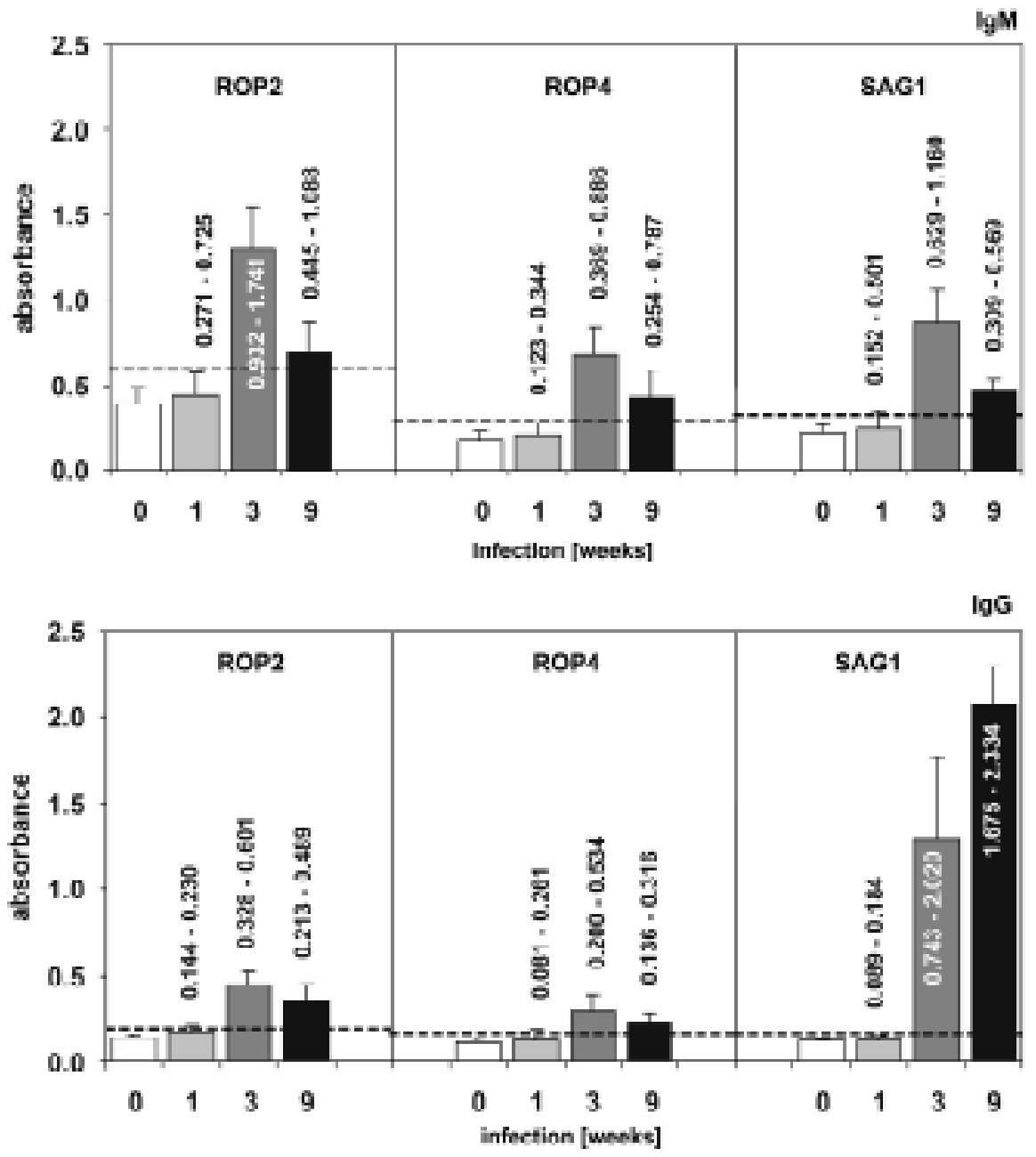

Fig. 1. Level of IgM and IgG antibodies against recombinant proteins ROP2, ROP4 and SAG1 in sera of mouce with experimental toxoplasmosis.

The cut-off values (- - ) are as follows: ROP2 IgM (0.587); ROP4 IgM (0.284); SAG1 IgM (0.314); ROP2 IgG (0.180); ROP4 IgG (0.162); SAG1 IgG (0.160). The absorbance ranges obtained in tests with sera of $T$. gondii infected animals are indicated above the columns.

with acute infections, might persist in the bloodstream of latently infected humans even for several years after primary invasion (Montoya, 2002) and many of the commercially available laboratory tests are susceptible to these clinically non-relevant IgM antibodies (Meek et al., 2001). The introduction to diagnostics of new well-defined recombinant antigens may be the solution and this study was aimed to determine the usefulness of recombinant ROP2 and ROP4 antigens for Toxoplasma invasion detection and phase discrimination in mouse experimental toxoplasmosis as a well-defined $T$. gondii infection model.

The results revealed a preferential synthesis of IgM antibodies directed against ROP proteins, mainly during acute toxoplasmosis, accompanied by smaller amounts of $\operatorname{IgG}$ antibodies. This phenomenon may result from the nature of ROP antigens and from the fact that they are released early in the invasion pro- cess only as antigens associated with host cell entry (Beckers et al., 1994; Carey et al., 2004). However Aubert et al. (2000) managed to detect specific antiROP2 IgM antibodies in nearly $15 \%$ of all human serum samples with an acute antibody profile and Jacobs et al. (1999) reported problems with the detection of IgM antibodies in human IgM-positive sera with the use of C-terminal fragment of ROP2 antigen. On the other hand, in our study the titers of IgM and IgG antibodies decreased during the latent phase of invasion, however both antibody classes were detected at a low level. Martin et al. (1998) reported that ROP2 antigen elicits a strong humoral response in humans, starting from an early stage of infection that involves both IgM and IgG antibodies, which in turn may be attributed to the fact, that ROP2 is detected in all T. gondii developmental stages (Sadak et al., 1988). The controversial results could be caused 
by various ROPs preparations which matched different fragments of ROP molecule and comprised or not individual conformational epitopes.

The performance of rhoptry proteins was compared to the major surface Toxoplasma antigen - SAG1. This antigen is used in some commercially available tests (Roux-Buisson et al., 2005) since it represents nearly $5 \%$ of parasite proteins and exhibits high antigenicity and immunogenicity expressed both by T-cell stimulation and specific antibody synthesis (Godard et al., 1994). What is more, SAG1 has been described as being useful for the detection of $\mathrm{IgG}$ antibodies in the sera of latently infected humans (Aubert et al., 2000; Pfrepper et al., 2005). In the present work the humoral response of $T$. gondii infected mice was characterized by a high anti-SAG1 antibody levels rising along with infection time. Similar observations were reported previously (Gatkowska et al., 2006) and these findings suggested that such a response is typical for mice, regardless of their genotype and innate susceptibility to $T$. gondii infection.

The results from the murine experimental model show that relatively high expressed IgM response to ROP antigens and SAG1 with considerable IgG antiSAG1 reactivity seems typical for acute toxoplasmosis, whereas a strong IgG reaction with SAG1 protein accompanied by low anti-ROP and anti-SAG1 IgM levels as well as trace amounts of anti-ROP IgG are characteristic for chronic toxoplasmosis. These results indicate that both rhoptry antigens - ROP2 and ROP4 induce humoral response which differs considerably depending on the phase of invasion. That suggests that both antigens may be considered for further investigation aimed at selection of antigens useful in toxoplasmosis diagnostics. The combination of rhoptry proteins with other $T$. gondii antigens may greatly improve their performance, as it has been shown previously that single-antigen (or even single-epitope) ELISAs may fail to recognize all positive samples and antigen epitope combination seems the most appropriate approach (Aubert et al., 2000; Beghetto et al., 2006).

\section{Acknowledgments}

M.Sc Roksana Jurga and M.Sc Magdalena Waleczko are thanked for their contribution to this study.

The study was supported by the Ministry of Sciences and Higher Education (grant N N302 3196 33) and grant of Łódź University.

\section{Literature}

Aubert D., G.T. Maine, I. Villena, J.C. Hunt, L. Howard, M. Sheu, S. Brojanac, L.E. Chovan, S.F. Nowlan and J.M. Pinon. 2000. Recombinant antigens to detect Toxoplasma gondii-specific immunoglobulin $\mathrm{G}$ and immunoglobulin $\mathrm{M}$ in human sera by enzyme immunoassay. J. Clin. Microbiol. 38: 1144-1150.
Beckers C.J.M., J.F. Dubremetz, O. Mercereau-Puijalon and K.A. Joiner. 1994. The Toxoplasma gondii rhoptry protein ROP2 is inserted into the parasitophorous vacuole membrane, surrounding the intracellular parasite, and is exposed to the host cell cytoplasm. J. Cell Biol. 127: 947-961.

Beghetto E., A. Spadoni, L. Bruno, W. Buffolano and N. Gargano. 2006. Chimeric antigens of Toxoplasma gondii: toward standardization of toxoplasmosis serodiagnosis using recombinant products. J. Clin. Microbiol. 44: 2133-2140.

Buffolano W., E. Beghetto, M. Del Pezzo, A. Spadoni, M. Di Cristina, E. Petersen and N. Gargano. 2005. Use of recombinant antigens for early postnatal diagnosis of congenital toxoplasmosis. J. Clin. Microbiol. 43: 5916-5924.

Carey K.L., A.M. Jongco, K. Kim and G.E. Ward. 2004. The Toxoplasma gondii rhoptry protein ROP4 is secreted into the parasitophorous vacuole and becomes phosphorylated in infected cells. Eukaryotic Cell. 3: 1320-1330.

de Souza Macre M., M. Pires, L.R. Meireles, S.O. Angel and H.F. de Andrade Jr. 2009. Serology using rROP2 antigen in the diagnostic of toxoplasmosis in pregnant women. Rev. Inst. Med. Trop. Sao Paulo. 51: 283-288.

Dziadek B., J. Gatkowska, A. Brzostek, J. Dziadek, K. Dzitko and H. Długońska. 2009. Toxoplasma gondii: The immunogenic and protective efficacy of recombinant ROP2 and ROP4 rhoptry proteins in murine experimental toxoplasmosis. Exp. Parasitol. 123: 81-89.

Ferrandiz J., C. Mercier, M. Wallon, S. Picot, MF. CesbronDelauw and F. Peyron. 2004. Limited value of assays using detection of immunoglobulin $G$ antibodies to the two recombinant dense granule antigens, GRA1 and GRA6 Nt of Toxoplasma gondii, for distinguishing between acute and chronic infections in pregnant women. Clin. Diagn. Lab. Immunol. 11: 1016-1021.

Gatkowska J., E. Hiszczyńska-Sawicka, J. Kur, L. Holec and H. Dlugońska. 2006. Toxoplasma gondii: An evaluation of diagnostic value of recombinant antigens in a murine model. Exp. Parasitol. 114: 220-227.

Godard I., J. Estaquier, L. Zenner, M. Bossus, C. Auriault, F. Darcy, H. Gras-Masse and A. Capron. 1994. Antigenicity and immunogenicity of P30-derived peptides in experimental models of toxoplasmosis. Mol. Immunol. 31: 1353-1363.

Jacobs, D., M. Vercammen and E. Saman. 1999. Evaluation of recombinant dense granule antigen 7 (GRA7) of Toxoplasma gondii for detection of immunoglobulin $\mathrm{G}$ antibodies and analysis of a major antigenic domain. Clin. Diagn. Lab. Immunol. 6: 24-29. Martin V., M. Arcavi, G. Santillan, M.R.R.Amendoeira, E. de Souza Neves, G. Griemberg, E. Guarnera, J.C. Garberi and S.O. Angel. 1998. Detection of human Toxoplasma-specific immunoglobulins $\mathrm{A}, \mathrm{M}$ and $\mathrm{G}$ with a recombinant Toxoplasma gondii Rop2 protein. Clin. Diagn. Lab. Immunol. 5: 627-631.

Mechain B., Y.J.F. Garin, F. Robert-Gangneux, J. DupouyCamet and F. Derouin. 2000. Lack of utility of specific immunoglobulin $\mathrm{G}$ antibody avidity for serodiagnosis of reactivated toxoplasmosis in immunocompromised patients. Clin. Diagn. Lab. Immunol. 7: 703-705.

Meek B., T. van Gool, H. Gilis and R. Peek. 2001. Dissecting the IgM antibody response during the acute and latent phase of toxoplasmosis. Diagn. Microbiol. Infect. Dis. 41: 131-137.

Meroni V., F. Genco, C. Tinelli, P. Lanzarini, L. Bollani, M. Stronati and E. Petersen. 2009. Spiramycin treatment of Toxoplasma gondii infection in pregnant women impairs the production and the avidity maturation of $T$. gondii-specific immunoglobulin G antibodies. Clin. Vaccine Immunol. 16: 1517-1520. Montoya J.G. 2002. Laboratory diagnosis of Toxoplasma gondii infection and toxoplasmosis. J. Infect. Dis. 185: S73-82. 
Montoya, J.G. and O. Liesenfeld. 2004. Toxoplasmosis. Lancet. 363: 1965-1976.

Nigro M., A. Gutierrez, A.M. Hoffer, M. Clemente, F. Kaufer, L. Carral, V. Martin, E.A. Guarnera and S.O. Angel. 2003. Evaluation of Toxoplasma gondii recombinant proteins for the diagnosis of recently acquired toxoplasmosis by an immunoglobulin G analysis. Diagn. Microbiol. Infect. Dis. 47: 609-613. Pfrepper KI., G. Enders, M. Gohl, D. Krczal, H. Hlobil, D. Wassenberg and E. Soutschek. 2005. Seroreactivity to and avidity for recombinant antigens in toxoplasmosis. Clin. Diagn. Lab. Immunol. 12: 977-982.

Pietkiewicz H., E. Hiszczyńska-Sawicka, J. Kur, E. Petersen, H.V. Nielsen, M. Stankiewicz, I. Andrzejewska and P. Myjak. 2004. Usefulness of Toxoplasma gondii-specific recombinant antigens in serodiagnosis of human toxoplasmosis. J. Clin. Microbiol. 42: 1779-1781.

Roux-Buisson N., H. Fricker-Hidalgo, A. Foussadier, D. Rolland, A.S. Suchel-Jambon, M.P. Brenier-Pinchart and H. Pelloux. 2005. Comparative analysis of the VIDAS Toxo IgG IV assay in the detection of antibodies to Toxoplasma gondii. Diagn. Microbiol. Infect. Dis. 53: 79-81.

Saavedra R., M.A. Becerril, C. Dubeaux, R. Lippens, M.J. de Vos, P. Herion and A. Bollen. 1996. Epitopes recognized by human $\mathrm{T}$ lymphocytes in the ROP2 protein antigen of Toxoplasma gondii. Infect. Immun. 64: 3858-3862.

Sadak A., Z. Taghy, B. Fortier and J.F. Dubremetz. 1988 Characterization of a family of rhoptry proteins of Toxoplasma gondii. Mol. Biochem. Parasitol. 29: 203-211.

Sambrook J. and D.W. Russell. 2001. Molecular cloning: a laboratory manual, $3^{\text {rd }}$ ed. Cold Spring Harbor, NY: Cold Spring Harbor Laboratory Press.

Sinai A.P. and K.A. Joiner. 2001. The Toxoplasma gondii protein ROP2 mediates host organelle association with the parasitophorous vacuole membrane. J. Cell Biol. 154: 95-108.

van Gelder P., F. Bosman, F. de Meuter, H. van Heuverswyn and P. Hérion. 1993. Serodiagnosis of toxoplasmosis by using a recombinant form of the 54-kilodalton rhoptry antigen expressed in Escherichia coli. J. Clin. Microbiol. 31: 9-15. 